\title{
Die Bestimmung der Umwandlungspunkte bei den Sulfaten, Molybdaten und Wolframaten des Natriums und Kaliums. Von
}

H. S. VAN KLOOSTER.

Mit 1 Figur im Text.

Bekanntlich zeigen mehrere anorganische Salze eine oder mehrere reversible polymorphe Umwandlungen. Mit der Feststellung der Umwandlungstemperaturen auf dilatometrischem, thermischem und optischem Wege sind eine ganze Anzahl von Forschern beschäftigt gewesen. Die meisten Untersuchungen dieser Art wurden bisher mittels der thermischen Methode angestellt, und zwar fast immer durch das Studium der Abkühlungserscheinungen. Dabei hat sich herausgestellt, daB die Gleichgewichtstemperaturen öfters überschritten werden, worauf z. B. schon im Jahre 1885-1886 Beldati und Romanese ${ }^{1}$ beim Studium des Kalium- und Ammoniumnitrats hingewiesen haben. Versuche, um durch Verringerung der Abkühlungsgeschwindigkeit in beliebigem Maßstabe eine bessere Annäherung an die wahren Gleichgewichtstemperaturen zu erhalten, sind bei den bisherigen Untersuchungen ausgeblieben. Es sind zwar Versuche gemacht worden, ${ }^{2}$ durch automatische Verringerung des Heizstromes (im Falle, daß elektrische Öfen benutzt wurden), anstatt der gewöhnlichen logarithmischen Ofenabkühlungskurven, gerade Kurven zu erhalten, was innerhalb nicht zu groBen Temperaturintervallen auch gelungen ist, aber das Problem einer in möglichst weiten Temperaturgrenzen in beliebigen Schranken zu haltender Abkühlungsgeschwindigkeit ist dadurch nicht gelöst worden.

Im folgenden habe ich deshalb versucht, mittels der am hiesigen Laboratorium üblichen Erhitzungs - und MeBmethoden, ${ }^{3}$ die haupt-

1 Atti. Ist. Veneto [6] 3 (1885), 653; 4 (1886), 1395.

${ }^{2}$ Prato, Zeitschr. phys. Chem. 55, 721; 58, 350; 63, 447 (1906-1908). Portevin, Revue de Métallurgie 5 (1908), 303.

${ }^{3}$ Siehe u. a. F. M. JAEGER, Eine Anleitung zur Ausführung exakter Messungen usw. Groningen, J. B. Wolters, 1913.

Z. anorg. Chem. Bd. 85 . 
sächlich durch $D_{A Y}$ und seine Mitarbeiter entwickelt wurden, die Umwandlungstemperaturen einiger hochschmelzender Salze festzulegen. Die Überlegenheit dieser Methode geht schon daraus hervor, daB man die Geschwindigkeit der Erhitzung nach Belieben regulieren und mit sehr geringen Schwankungen konstant erhalten kann, und zwar in beliebigen Temperaturgrenzen. - Nun ist nicht zu leugnen, daB auch bei der Erhitzung Überschreitungen der Umwandlungstemperaturen stattfinden können und auch wirklich manchmal vorkommen. ${ }^{1}$ Namentlich geschieht dies bei erstmaligem Erhitzen, wenn die Umwandlungswärme ziemlich bedeutend ist. Die Überhitzung macht sich dann bemerkbar, entweder durch eine sichtbare Überschreitung der. Umwandlungstemperatur, gekennzeichnet durch zeitweises Sinken der Temperatur, oder aber durch eine Verschleppung, wobei die Umwandlung nicht bei einer bestimmten Temperatur stattfindet, sondern sich über ein mehr oder weniger großes Temperaturintervall erstreckt. Wenn man aber in diesem Falle nach dem Vorschlag von Bakhuis Roozeвоом ${ }^{2}$ gleich wieder abkühlt, bis die Rückverwandlung vollständig eingetreten ist, und von neuem erhitzt, findet in der Regel die Umwandlung bei einer bestimmten Temperatur statt, ohne jegliche Überschreitung. Daß die gefundene Temperatur die richtige ist, läBt sich noch näher verifizieren durch nochmalige Wiederholung des Versuches, wobei man die Erhitzungsgeschwindigkeit in bestimmtem Maße verringert. Sollte die gefundene Temperatur nicht die richtige gewesen sein, so müBte dann die Umwandlung bei noch niedrigerer Temperatur stattfinden. - Es hat sich gezeigt, daß die Umwandlungstemperaturen bei der Erhitzung viel weniger überschritten werden als bei der Ablühlung. Bei kleiner Umwandlungswärme findet eine Überschreitung sogar nur ausnahmsweise statt. Die Umwandlung äußert sich dann durch einen Knick in der Erhitzungskurve, der bei jeder Erhitzungsgeschwindigkeit (natürlich innerhalb gewissen Grenzen, z. B. von 20-70 Mikrovolt [abgekürzt M.V.] entsprechend $2-7^{\circ}$ pro Minute) an derselben Stelle zurückgefunden wird. - Das Kriterium für die wahren Umwandlungstemperaturen ist also darin gesucht worden, daß dieselben sich unabbängig zeigen von der Erhitzungsgeschwindigkeit.

Als Untersuchungsobjekte habe ich die Sulfate, Molybdate und

1 Siehe u. a. Bellati und Romanese, l. c.

${ }^{2}$ Die heterogenen Gleichgewichte, Bd. I, 1, 114. 
Wolframate des Natriums und Kaliums gewählt, weil diese Salze alle mindestens dimorph sind und die Umwandlungstemperaturen nur mit großer Annäherung bekannt sind. Die Salze wurden auf zweierlei Weise thermisch untersucht (im folgenden kurz mit $\mathrm{A}$ und $B$ angedeutet). Zuerst wurden sie in einem schwach konischen Platintiegel von etwa $12 \mathrm{qcm}$ Inhalt, der bis zur Hälfte oder zu $2 / 3$ der Höhe gefüllt wurde, geschmolzen. Als Ofen wurde in diesem Fall ein groBer Nichrombandofen mit Innenwickelung benutzt, der noch, weil die Temperaturen alle unter $1200^{\circ}$ lagen, mit einem Innenzylinder aus Hartporzellan versehen war. Oben und unten wurde der Ofen mit Porzellan- und Asbestscheiben abgedeckt. Der Ofen war dem hier üblichen Typus der Platinwiderstandsöfen nachgebildet. ${ }^{1}$ Der Tiegel stand auf einer Porzellanscheibe, die von einem MaRQUaRdT schen Schutzrohr (10 mm Durchmesser) getragen wurde. Die Einstellhöhe des Tiegels im kleinen Gebiet der konstanten Temperatur war durch Vorversuche festgestellt.

Zweitens wurden die Salze in einem groBen Platindrahtofen mit Innenwickelung erhitzt. Auch hier wurde vorher das Gebiet der Temperaturkonstanz, das sich auf ein Gebiet von ca. $4 \mathrm{~cm}$ erstreckte, aufgesucht. In dieses Gebiet wurde ein ganz kleiner zylindrischer Platintiegel von ca. $10 \mathrm{~mm}$ Durchmesser und $1 \frac{1}{2} \mathrm{qcm}$ Inhalt (zu etwa $2 / 3$ mit dem geschmolzenen Salz angefüllt) hineingehängt. ${ }^{2}$

Die Erhitzung der Öfen geschah mit Gleichstrom von 110 Volt Spannung und die innerhalb weiter Grenzen durchführbare Regnlierung der Erhitzungsgeschwindigkeit mittels eines $11.1 \Omega$ RoHstratTschen Dekadenvorschaltwiderstandes.

Die benutzten Thermoelemente waren die jetzt allgemein gebräuchlichen aus $\mathrm{Pt}-\mathrm{PtRh}(10 \%)$ in der Stärke von $0.4 \mathrm{~mm}$. Die Lage der zweiten Lötstelle des Massenelements (die erste wurde im Eiskessel auf $0^{\circ}$ gehalten) war immer zentral in der Mitte, etwa 4-5 mm vom Boden des Tiegels entfernt. Abweichungen von der Zentralstellung können mitunter schon beträchtliche Fehler herbeiführen. Eine zu hohe Lage der Lötstelle veranlaßt unmittelbar eine bedeutende Diskrepanz zwischen Schmelz- und Erstarrungspunkt (die bei leicht kristallisierbaren Salzen, wie z. B. das $\mathrm{Na}_{2} \mathrm{WO}_{4}$ praktisch zusammenfallen), und zwar wird dadurch namentlich der Schmelzpunkt ganz verwischt und zu hoch gefunden.

${ }^{1}$ Siehe JaEgER, l. c. S. 36 u. f.

${ }^{2} J_{\text {AEGER, }}$ l. c. S. 59. 
Die Temperatur wurde auf einem WoLFsschen Dreidekadenkompensationsbank in Mikrovolts abgelesen, wobei immer bis auf 200 M.V. kompensiert wurde. Der jeweilige Restbetrag wurde mittels eines empfindlichen Galvanometers des Ayrton-Mathertypus bestimmt, wobei $1 \mathrm{~mm}$ der Skala 2 M.V. entsprach. Bei der Erhitzung wurde, zwecks Kontrollierung des regelmäßigen Anstiegs der Ofentemperatur ein zweites Thermoelement, in einem Schutzrohr eingeschlossen, neben dem Schmelztiegel in kleiner Entfernung angebracht, und die Temperatur des Ofens und der Schmelze abwechselnd (mit Hilfe eines Umschalters) jede halbe Minute abgelesen.

Die Eichung des Massenelements geschah wiederholt im Laufe der Untersuchung durch Schmelzpunktsbestimmung einiger reinen Metalle und Salze, die von $D_{A X}$ und seinen Mitarbeitern genau bestimmt worden sind. Als solche wurden gewählt: Lithiummetasilikat, Natriumsulfat, Zink, Cadmium, deren Schmelzpunkte folgende Werte haben:

$$
\begin{aligned}
& \mathrm{Li}_{2} \mathrm{SiO}_{3}: 1201^{0} \pm 1^{0} \text { Sosman (1), }{ }^{1} \mathrm{~J}_{\text {AEger }}(2) \\
& \mathrm{Na}_{2} \mathrm{SO}_{4}: 884^{\circ} \pm 1^{\circ} \text { White (3), DaY und Sogman (4) } \\
& \text { Zink: } \quad 418^{\circ} \\
& \text { Cadmium: } 320^{\circ} \\
& \text { Day und Sosman (1). }
\end{aligned}
$$

Die Eichung mittels Metallen wurde möglichst beschränkt, um eventuelle Infektion der Elemente vorzubeugen.

$\mathrm{Na}_{2} \mathrm{SO}_{4}$. - Was das Natriumsulfat anbelangt, so kann ich mich ganz kurz fassen, weil das Salz bereits eingehend ron WHITE (3) untersucht worden ist. Eine Übersicht tuber die von verschiedenen Forschern gefundenen Schmelz- und Umwandlungspunkte (nur die neueren mittels Thermoelementen bestimmten Daten sind angegeben) gibt folgende Tabelle.

\section{(S. Tabelle 1, S. 53.)}

Wie ersichtlich liefert der Vergleich wenig übereinstimmende Resultate. Beim Schmelzpunkt liegen die extremen Werte nicht weniger als $11^{\circ}$ von dem Mittelwert ab. Das ,Mittel“ hat übrigens hier sowohl als in anderen Fällen nur eine geringe Bedeutung, weil dabei die Beobachtungen alle als gleichwertig angesehen wurden, was wohl sicher nicht der Fall sein mag. Beim Umwandlungspunkt liegen die extremen Werte sogar noch $14^{0}$ auseinander.

${ }_{1}$ Die eingeklammerten Zahlen hinter den Autorennamen beziehen sich auf die Literaturzusammenstellung am Schluß der Abhandlung. 
Tabelle 1.

\begin{tabular}{|c|c|c|}
\hline Beobachter & $\begin{array}{l}\text { Schmelzpunkt } \\
\text { in } 0\end{array}$ & $\begin{array}{l}\text { Umwandlungspunkt } \\
\text { in } 0\end{array}$ \\
\hline 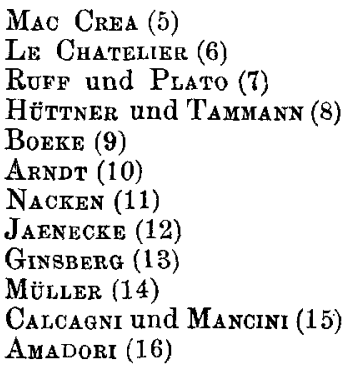 & $\begin{array}{l}\text { ca. } 883 \\
875 \\
880 \\
897 \\
888 \\
883 \\
883 \\
897 \\
883.5 \\
881 \\
887 \\
892\end{array}$ & $\begin{array}{l}- \\
\overline{-} \\
235 \\
239 \\
- \\
234 \\
225 \\
232 \\
233 \\
234 \\
238\end{array}$ \\
\hline $\begin{array}{r}\text { Mit } \\
\text { WhITE }\end{array}$ & $\begin{array}{l}886 \pm 11 \\
884 \pm 1\end{array}$ & $\begin{array}{c}234 \pm 7 \\
-\end{array}$ \\
\hline
\end{tabular}

Auffallend sind die Differenzen, wenn man bedenkt, daB die meisten Forscher ihre Angaben (durch Eichung der Thermoelemente mittels reinen KaHLBadmschen Metallen) auf die Skala der Reichsanstalt bezogen haben, welche unterhalb $1000^{\circ}$ nur um höchstens $1^{0}$ von der neueren WASHINGTON schen abweicht.

Ein wichtiger Punkt ist die Reinheit der benutzten Präparate wie White (3, l. c. S. 459) zuerst klar gezeigt hat. Ich habe mich überzeugen können, daß die Form der Schmelzkurven sich durch geringe Verunreinigungen, wie sie schon durch wiederholte Benutzung des Materials sogar unabsichtlich in dem Salz enthalten sein können, sofort ändert. Es zeigt sich dann, daB die Temperatur sich nicht 2-3 Minuten auf dem Schmelzpunkt hält (Tiegel B, 2-2.5 g Substanz, Erhitzungsgeschwindigkeit 40 M.V. pro Min.), sondern sich stetig ändert, und nach vollständigem Schmelzen sehr rasch emporschnellt.

Auch die Umwandlungstemperatur wird durch geringe Beimischungen schon merklich erniedrigt. Es empfiehlt sich dabei, von einer größeren Menge reinen Salzes, zwecks Eichung, jedesmal eine neue Portion zu nehmen, was bei der geringen Menge, die man für eine Bestimmung nach der hier gefolgten Methode braucht, keine Schwierigkeiten machen kann.

Das $\mathrm{Na}_{2} \mathrm{SO}_{4}$ zeigt thermisch nur eine Umwandlung, die sowohl bei der Abkühlung wie auch bei der Erhitzung leicht um einige Grade überschritten werden kann. Operiert man aber nach den eingangs erwähnten Methoden, so läßt sich die Umwandlung mit einer Genauigkeit von $\pm 1^{\circ}$ bei $241^{\circ}$ festlegen. 
$\mathrm{Na}_{2} \mathrm{MOO}_{4} \cdot$ - Als Ausgangsmaterial wurde das $\mathrm{K}_{\mathrm{AHLBATM}}$ sche Dihydrat benutzt. $17.0031 \mathrm{~g}$ Substanz verloren bei vorsichtiger Er. wärmung bis zum Schmelzen des wasserfreien Salzes erhitzt $2.5413 \mathrm{~g}$ Wasser. Berechnet für $\mathrm{Na}_{2} \mathrm{MoO}_{4} \cdot 2$ aq $\mathrm{H}_{2} \mathrm{O}: 14.89 \%$, gefunden: $14.94 \%$.

Qualitativ konnte kein Sulfat und Halogen nur spurenweise nachgewiesen werden.

Dieses Salz bildet ein sehr interessantes Untersuchungsobjekt, weil es, wie BoEke (9) zuerst gefunden hat, nicht weniger als drei Umwandlungen in festem Zustande zeigt, also tetramorph ist. Im folgenden gebe ich die Daten (unkorrigiert), wie sie aus den erhaltenen Erhitzungskurven abgeleitet wurden. Die Kurven sind schon an anderer Stelle ${ }^{l}$ reproduziert worden; die dort beigeschriebenen Zahlen sind, wegen der damals noch nicht sicheren Korrektur des Thermo-elements nicht ganz richtig angegeben worden.

Zur Untersuchung gelangen ca. $15 \mathrm{~g}$ wasserfreies Salz, Tiegeltypus A. Die Werte sind angegeben in M.V., der sichtbare Betrag einer Überhitzung ist durch die eingeklammerten Zahlen angegeben.

Tabelle 2.

\begin{tabular}{|c|c|c|c|c|c|c|c|}
\hline 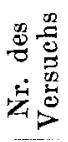 & $\begin{array}{l}\text { Erhitzung } \\
\text { yon-bis- } \\
\text { in M.V. }\end{array}$ & $\begin{array}{l}\text { Dauer des } \\
\text { Versuchs } \\
\text { in Min. }\end{array}$ & $\begin{array}{c}\text { Mittl.Erh.- } \\
\text { Gesehwin- } \\
\text { digkeit } \\
\text { M.V.i.Min. }\end{array}$ & $\begin{array}{c}\text { Umwand- } \\
\text { lung I }\end{array}$ & $\begin{array}{l}\text { Umwand- } \\
\text { lung II }\end{array}$ & $\begin{array}{l}\text { Umwand- } \\
\text { lung III }\end{array}$ & $\begin{array}{l}\text { Sehmelz- } \\
\text { punkt }\end{array}$ \\
\hline $\begin{array}{l}1 \\
2 \\
3\end{array}$ & $\begin{array}{l}3200-7000 \\
2900-6800 \\
3300-6400\end{array}$ & $\begin{array}{r}42 \\
99 \\
150\end{array}$ & $\begin{array}{l}70 \\
40 \\
20\end{array}$ & $\begin{array}{l}3758(20) \\
3700(6) \\
3700(6)\end{array}$ & $\begin{array}{l}5172 \\
5164 \\
5166\end{array}$ & $\begin{array}{l}5664 \\
5666 \\
5664\end{array}$ & $\begin{array}{l}6148 \\
6144 \\
6156\end{array}$ \\
\hline \multicolumn{3}{|c|}{ Wabrscheinlicher } & er Wert: & 3700 & $5167 \pm 4$ & $5665 \pm 1$ & $6149 \pm 7$ \\
\hline
\end{tabular}

Zum Vergleich gebe ich daneben die von Herrn Dr. A. Šrmek bei derselben Versuchsanordnung und ungefähr derselben Beschickung und mit dem nämlichen Thermoelement 5 Wochen später erhaltenen Zahlen:

Tabelle 3.

\begin{tabular}{|c|c|c|c|c|c|c|c|}
\hline 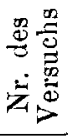 & $\begin{array}{c}\text { Erbitzung } \\
\text { von-bis- } \\
\text { in M.-V. }\end{array}$ & $\begin{array}{l}\text { Dauer des } \\
\text { Versuchs } \\
\text { in Min. }\end{array}$ & $\begin{array}{c}\text { Mittl.Erh.- } \\
\text { Geschwin- } \\
\text { digkeit } \\
\text { M.V.i.Min. }\end{array}$ & $\begin{array}{l}\text { Umuand- } \\
\text { lung I }\end{array}$ & $\begin{array}{l}\text { Unwand- } \\
\text { lung II }\end{array}$ & $\begin{array}{l}\text { Unwand- } \\
\text { lung III }\end{array}$ & $\begin{array}{l}\text { Schmelz- } \\
\text { punkt }\end{array}$ \\
\hline 1 & $|3200-7000|$ & 61 & 60 & 3726 & 5162 & 5666 & 6140 \\
\hline 2 & $400-7000$ & 176 & 40 & 3720 & 5160 & 5676 & 6150 \\
\hline 3 & $|3000-6600|$ & 175 & 20 & 3700 & 5166 & 5670 & 6138 \\
\hline \multicolumn{3}{|c|}{ Wahrscheinliche } & er Wert: & 3700 & $5163 \pm 3$ & $5671 \pm 5$ & $6143 \pm 7$ \\
\hline
\end{tabular}


Es wurden darauf noch zwei Versuche mit einer kleinen Beschickung ron $3.5 \mathrm{~g}$ (Tiegeltypus $\mathrm{B}$ ) ausgeführt mit nahezu gleichem Resultat (Geschwindigkeit der Erhitzung in beiden Fällen etwa 40 M.V. pro Minute). Das Resultat dieser drei Versuchsreihen ist in der folgenden Tabelle zusammengefaBt (korrigierte Angaben).

Tabelle 4.

\begin{tabular}{|c|c|c|c|c|c|}
\hline Beobachter & $\begin{array}{l}\text { Tiegel- } \\
\text { typus }\end{array}$ & $\begin{array}{l}\text { Umwand- } \\
\text { lung I }\end{array}$ & $\begin{array}{l}\text { Umwand- } \\
\text { lung II }\end{array}$ & $\begin{array}{l}\text { Umwand- } \\
\text { lung III }\end{array}$ & $\begin{array}{l}\text { Schmelz- } \\
\text { punkt }\end{array}$ \\
\hline $\begin{array}{l}\text { VAN KLOOSTER } \\
\text { SIMEK } \\
\text { VAN KLOOSTER }\end{array}$ & $\begin{array}{l}\mathrm{A} \\
\mathrm{A} \\
\mathrm{B}\end{array}$ & $\begin{array}{ll}3690 & (6) \\
3690 & (0) \\
3704 & (44)\end{array}$ & $\begin{array}{l}5149 \pm 5 \\
5145 \pm 3 \\
5142 \pm 4\end{array}$ & $\begin{array}{l}5645 \pm 1 \\
5651 \pm 5 \\
5639 \pm 2\end{array}$ & $\begin{array}{l}6127 \pm 7 \\
6121 \pm 7 \\
6126 \pm 3\end{array}$ \\
\hline & Mittel & 3690 & 5145 & 5645 & 6125 \\
\hline
\end{tabular}

Wie aus allen diesen Zahlen hervorgeht, erreicht bei diesen beiden ganz verschiedenen Versuchsanordnungen und Erhitzungsgeschwindigkeiten die Differenz zwischen den extremen Werten selbst im ungünstigsten Falle kaum 20 M.V. (etwa $2^{0}$ ). Man kann daher die Reproduzierbarkeit bis auf $\pm 0.5-1^{\circ}$ genau einschätzen.

Nur bei der Umwandlung I, die durch eine im Vergleich mit den anderen, sehr große Wärmeabsorption bei der Erhitzung charakterisiert ist, ist der EinfluB der Erhitzungsgeschwindigkeit deutlich erkennbar. Die niedrigsten Temperaturen stimmen aber bei der ersten und der zweiten Versuchsreihe vollkommen überein.

Umgerechnet auf die Skala von $D_{A Y}$ und Sosman (1) findet man folgende Werte in ${ }^{0}$ :

Umwandlung I Umwandlung II Umwandlung III Schmelzpunkt $\begin{array}{llll}445^{\circ} & 592^{\circ} & 640^{\circ} & 687^{\circ}\end{array}$

Zum Vergleich sind hier unten die von früheren Forschern, so wie von mir die nach der Abkühlungsmethode erhaltenen Temperaturen angegeben.

Tabelle 5.

\begin{tabular}{|c|c|c|c|c|}
\hline Beobachter & $\begin{array}{l}\text { Umwand- } \\
\text { lung I } \\
\text { in }{ }^{0}\end{array}$ & $\begin{array}{l}\text { Umwand- } \\
\text { lung II } \\
\text { in }{ }^{0}\end{array}$ & $\begin{array}{l}\text { Umwand- } \\
\text { lung III } \\
\text { in }{ }^{0}\end{array}$ & $\begin{array}{c}\text { Schmelz- } \\
\text { punkt } \\
\text { in }{ }^{0}\end{array}$ \\
\hline Hüttaner und Tammann (8) & $410-380$ & - & $620-605$ & 692 \\
\hline BOEKe (9) & 431 & 587 & 619 & 692 \\
\hline Groschuff (17) & 408 & 575 & 609 & 686 \\
\hline van Klooster (diese Arbeit) & 397 & 580 & 626 & 685 \\
\hline
\end{tabular}


Man erkennt wieder sofort: soviele Beobachter, ebensoviele verschiedene Werte; die Übereinstimmung der Schmelzpunkte ist noch leidlich, aber bei den verschiedenen Umwandlungen treten Differenzen von $15-30^{\circ}$ auf.

Es ist also ohne weiteres klar, daB es nicht statthaft ist, von. einer abnormen Erniedrigung der Umwandlungstemperatur I durch Zusatz von geringen Mengen Natriumsulfat zu sprechen, wie es BOEKE ${ }^{1}$ auf Grund seiner Abkühlungsversuche tun zu dürfen glaubte. Die Sache ist nämlich, daB die Umwandlungsverzögerung, die schon ohne Zusatz von Fremdstoffen auftritt, noch bedeutend durch Zusatz von anderen Salzen vergrößert wird.

$\mathrm{Na}_{2} \mathrm{WO}_{4} \cdot$ - Die Unzulänglichkeit der Abkühlungsmethode für die Bestimmung von Umwandlungstemperaturen tritt in unzweideutiger Weise ans Licht bei der Untersuchung gerade dieses Salzes, denn hier wird das Vorhandensein einer Umwandlung vorgetäuscht, die in Wirklichkeit gar nicht existiert. Nach den bisherigen Untersuchungen ist dieses Salz nämlich trimorph, wie aus folgender Tabelle ersichtlich ist.

Tabelle 6.

\begin{tabular}{|c|c|c|c|}
\hline Beobachter & $\begin{array}{l}\text { Umwandlung I } \\
\text { in }\end{array}$ & $\begin{array}{l}\text { Umwandlg. If } \\
\text { in }{ }^{0}\end{array}$ & $\begin{array}{l}\text { Schmelzpunkt } \\
\text { in }{ }^{0}\end{array}$ \\
\hline HütTNER und TamuanN (8) & 570 & - & $698(690$ ? l. c. S. 219) \\
\hline Bonke (9) & 564 & 588 & 698 \\
\hline Parravano (18) & 568 & 585 & 698 \\
\hline VAN KLooster (19) & 572 & 589 & 700 \\
\hline
\end{tabular}

Überraschend ist hier die große Übereinstimmung der Schmelzpunkte; auch für die Umwandlung II stimmen die Zahlen innerhalb $4^{0}$ überein, für die Umwandlung $I$ ist die maximale Differenz aber schon $8^{0}$.

Als Material für diese Untersuchung diente das Kahlbaumsche Dihydrat.

$16.182 \mathrm{~g}$ verloren bei vorsichtiger Erhitzung bis zum Schmelzen des Salzes erhitzt $1.7684 \mathrm{~g}$ Wasser.

Ber. für $\mathrm{Na}_{2} \mathrm{WO}_{4}, 2$ aq $\mathrm{H}_{2} \mathrm{O}: 10.92 \%$. Gef.: $10.93 \%$.

Auch die Resultate der thermischen Untersuchung waren in Einklang mit der vollkommenen Reinheit des Präparates. Zur besseren Orientierung über die hier vorliegenden Verhältnisse repro-

${ }^{1}$ Zeitschr. phys. Chem. $\mathbf{5 6}$ (1906), 686. 
Die Bestimmung der Umwandlungspunkte bei den Sulfaten usw. 57 duziere ich hier (Fig. 1) einige typische Erhitzungs- und Abkühlungskurven.

Die Kurve Ia (nebst zugehöriger Ofenkurve) wurde erhalten bei Benutzung des Tiegels $B$ mit $4 \mathrm{~g}$ Substanz.

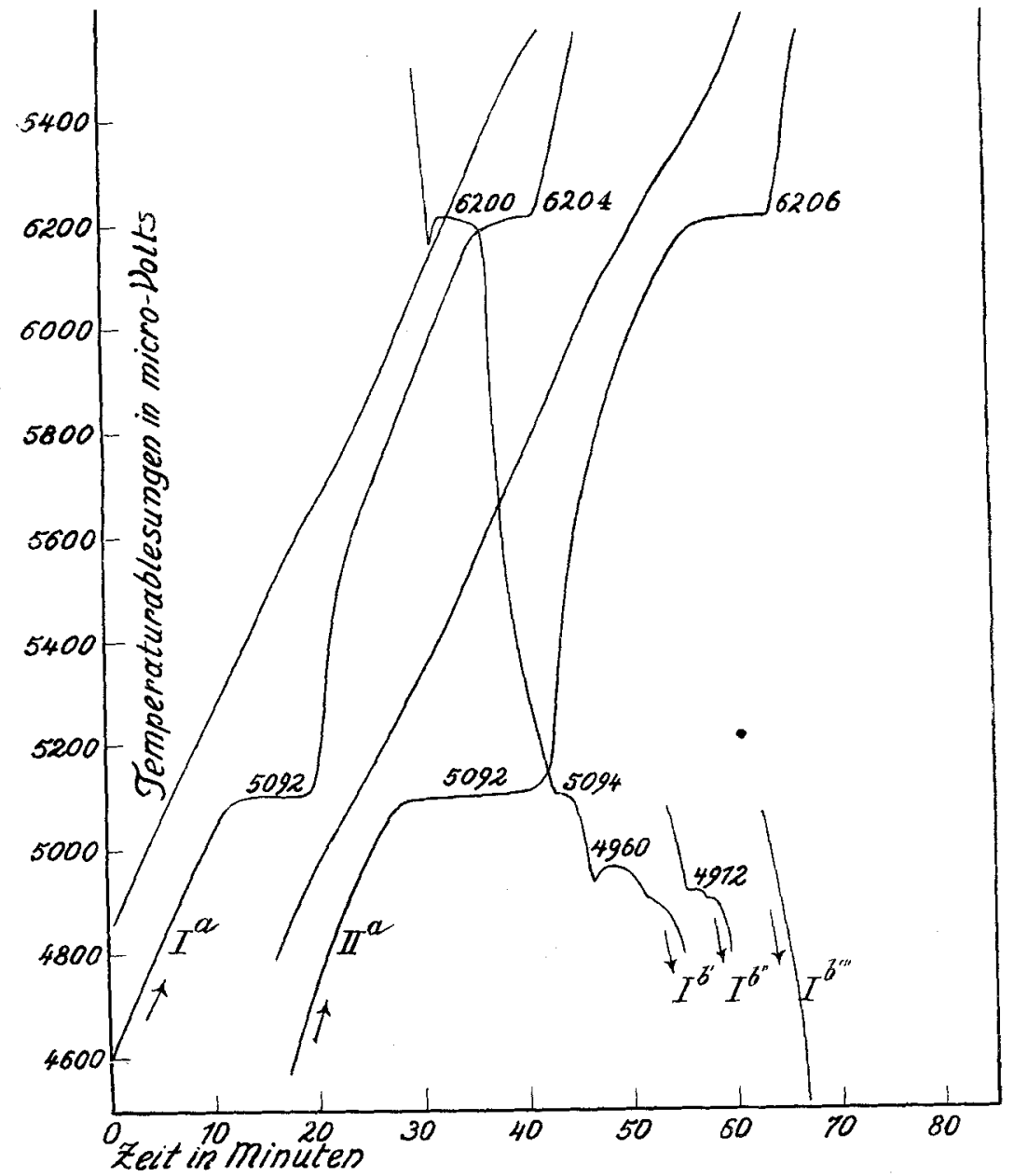

Fig. 1. Erhitzungs- und Abkühlungskurven von reinem Natriumwolframat.

$I b^{\prime}$ ist die zugehörige Abkühlungskurve (nach Ausschalten des Heizstromes erhalten).

II a ist eine Erhitzungskurve von $22 \mathrm{~g}$ Salzim Tiegel A geschmolzen.

Die Übereinstimmung zwischen I a und II a ist vollkommen; nur die Abrundung gegen das Ende der Umwandlung ist im zweiten Fall natürlich etwas größer, daher ist auch der Tiegel B hier vor- 
zuziehen, um so mehr weil dann die Massenkurve sich eher wieder dem Lauf der Ofenkurve anschmiegt.

$I b^{\prime}$ zeigt überzeugend, wie selbst nach einer Unterkühlung von 50 M.V. (ca. $5^{\circ}$ ) die Temperatur doch woch bis auf den Schmelzpunkt $694^{\circ}$ heraufschnellt. Genau bei derselben Temperatur, wo bei der Erhitzung eine Umwandlung auftrat, zeigt sich auch hier ein Effekt; die Temperatur sinkt aber bald, bis, etwa $13^{\circ}$ niedriger, nach vorhergehender Unterkühlung eine weitere Wärmetönung bemerkbar wird. Man könnte sogar noch einen um $6^{\circ}$ niedrigeren Effekt annehmen, obgleich dergleichen geringe Effekte in ähnlichen Fällen auch von anderen Autoren (vgl. BoEkE 9, 1. c. S. 360) nicht als Umwandlungen entsprechend qualifiziert sind. Um zu zeigen, daB diese zwei Effekte bloß durch Verzögerungen in der Umwandlung hervorgerufen sind, wurde das Salz (im Tiegel B) abkühlen gelassen bis gerade unterhalb der Umwandlungstemperatur $\left(587^{\circ}\right)$ und dann während einer halben Stunde bei $586 \pm 1^{\circ}$ konstant erhalten. Darauf wurde der Heizstrom abgestellt und abgekühlt. Das Resultat gibt die Kurre I b", die einen noch merkbaren Wärmeeffekt anzeigt, bei ca. $570^{\circ}$. Nach rollständiger Umwandlung wurde von neuem erhitzt, und zwar so, dab die Temperatur bis $583^{\circ}$ wieder gestiegen, während 15 Minuten innerhalb $0.3^{0}$ konstant blieb. Eine erneute Abkühlung ergab dann die glatte Kurve $I b^{\prime \prime}$. Hieraus geht mit Sicherheit hervor, daB nur eine einzige Umwandlung bei $587^{\circ}$ existiert.

Es fragt sich, wie man die erhaltenen Abkühlungskurven interpretieren kann. Man könnte hier versuchen die Tammans schen Ansich ten von Kernzahlbild ung und Kristallisationsgeschwindigkeitmutatis mutandis auszudehnen auf das Gleichgewicht Fest-Fest, wie dies bereits von $K_{\text {RUYT }^{1}}$ für die Umwandlungen der cis-Zimtsäuren geschehen ist. Zwar sind die Verhältnisse nicht ganz analog, da bekanntlich das Gleichgewicht Flüssig-Fest fast ausnahmslos nur in einer Richtung überschritten wird und das Gleichgewicht Fest-Fest bisweilen in beiden Richtungen, was der Auffassung entsprechen würde, da $B$ der Übergang von einem geordneten Molekularaggregat zu einem zweiten mehr Zeit beansprucht als von einem geordneten zu einem ungeordneten Zustand. Trotzdem geht beim Natriumwolframat die Analogie ziemlich weit. Denn die Umwandlungstemperatur wird bei der Erhitzung anscheinend gar nicht überschritten, während beim Abkühlen eine beträchtliche Überschreitung eintritt.

1 Berl. Ber. 44 (1912), 3108. 
Ähnliche Erscheinungen wurden früher bei der Abkühlung von Kalium- und Natriummetaboratschmelzen beobachtet. Bei diesen Salzen passiert es nämlich manchmal, daß die beginnende Erstarrung sich durch einen deutlichen Knick in der Abkühlungskurve kennbar macht, die Temperatur sinkt aber allmählich, um dann nach einigen Minuten wieder in die Höhe zu steigen. Tritt aber anfänglich eine bedeutende Unterkühlung ein, so schnellt die Temperatur plötzlich auf den Schmelzpunkt empor, wo er sich längere Zeit hält, um dann wieder zu sinken. Diese Sachlage läbt sich dadurch erklären, daß schon wenig unterhalb des Schmelzpunktes eine merkliche Kernzahlbildung auftritt, ohne daB die Kristallisationsgeschwindigkeit dabei eine bedeutende ist. Bleibt die Bildung von Kernen unter Umständen aus, so entstehen mehrere Grade unterhalb des Schmelzpunktes plötzlich schnell wachsende Kristallindividuen.

Beim Umwandlungspunkt des $\mathrm{Na}_{2} \mathrm{WO}_{4}$ müBte man nun annehmen, daB die „Umwandlungskernbildung“ beim Abkühlen immer genau bei $587^{\circ}$ eintritt, die Wachstumsgeschwindigkeit aber erst etwa $15^{0}$ später ihren maximalen Wert erreicht. Warum aber bei der Erhitzung die Rückverwandlung so glatt verläuft, mub dahingestellt bleiben, bis man sich darüber klar geworden ist, weshalb im allgemeinen bei Phasenverschiebungen gerade die Wärmeabsorption sich so viel leichter vollziehen läBt als die Wärmeabgabe.

$\mathbf{K}_{2} \mathbf{S O}_{\mathbf{4}} \cdot$ - Übergehend zu den Kaliumsalzen konnte man beim reinen Kaliumsulfat, durch zweimalige Umkristallisation des Ausgangsmaterials erbalten, eine gute Übereinstimmung der Resultate bei Benutzung der beiden Tiegeltypen beobachten, wie folgende Tabelle zeigt. Die Erhitzungsgeschwindigkeit variierte hier von 40-50 M.V. pro Minute. Die Schmelzpunkte sind auf den Kurven sehr scharf; die Umwandlung machte sich sowohl bei der Erbitzung als bei der Abkühlung nur durch eine geringe Verzögerung in dem geradlinigen Kurvenlauf kennbar.

Tabelle 7.

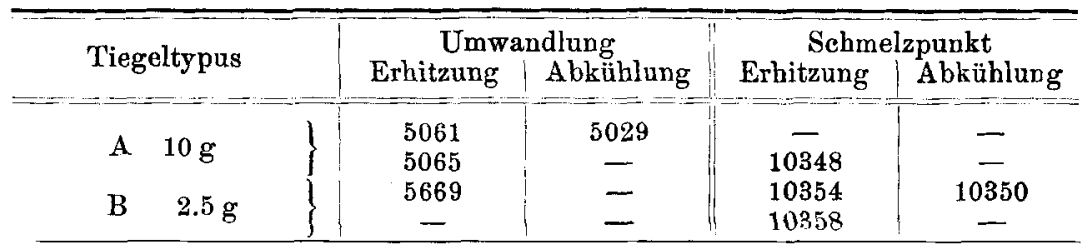

Im Mittel 506ई̃ \pm 4 M.V.

$10353 \pm 5$ M.V. 
Die Differenz zwischen den Umwandlungstemperaturen bei der Erhitzung und bei der Abkühlung beträgt hier nur ca. 30 M.V. $=3^{\circ}$. Nimmt man eine sehr geringe Überschreitung in beiden Richtungen an, so ist der wahrscheinliche Wert ungefähr 5046 M.V. oder $582^{\circ}$. Bekanntlich hat zuerst MaLuand (20) auf optischem Wege die Umwandlung gefunden und sie zwischen 600 und $650^{\circ}$ festgelegt. Später hat Le Chatelier (21) die Temperaturgrenzen thermisch bestimmt zwischen 530 und $580^{\circ}$. Nach neueren Untersuchungen, die sich aber wieder nur auf Abkühlungskurven stützen, liegt sie zwischen 580 und $599^{\circ}$. Ich gebe in der folgenden Tabelle die Angaben aus den letzten 10 Jahren, nebst den gefundenen Schmelz- (eigentlich Erstarrungs-)punkten:

Tabelle 8.

\begin{tabular}{|c|c|c|}
\hline Beobachter & Schmelzpunist in ${ }^{\circ}$ & Umwandlungsp. in ${ }^{\circ}$ \\
\hline RefF und Plato (7) & 1050 & - \\
\hline Hüttner und Tammana (8) & 1074 & 587 \\
\hline NaCkeN $(11)$ & 1076 & 595 \\
\hline $\mathrm{K}_{\text {ARANDEEFF }}(22)$ & 1074 & 599 \\
\hline MULLER (14) & 1057 & 580 \\
\hline GroschuFf (17) & 1072 & 586 \\
\hline CalcagnI $(23)$ & 1066 & 590 \\
\hline Amadori (16) & 1066 & 583 \\
\hline Amadori (24) & 1070 & 585 \\
\hline & $\begin{array}{ll}\text { ittel : } & 1067 \pm 13 \\
\text { beit: } & 1067 \pm 0.5\end{array}$ & $\begin{array}{l}588 \pm 10 \\
582 \pm 1.5\end{array}$ \\
\hline
\end{tabular}

Merkwürdigerweise stimmt das Mittel sämtlicher Schmelzpunktbestimmungen, trotzdem die extremen Werte $26^{\circ}$ auseinander liegen genau mit dem von mir gefundenen Wert überein; allerdings ist die Abweichung rom Mittelwert etwa $30 \mathrm{mal}$ größer.

$\mathbf{K}_{2} \mathbf{M o O}_{4}$. - Das Kaliummolybdat und das - Wolframat sind gekennzeichnet durch Umwandlungen, die, obwohl bei Temperaturerniedrigung daran erkennbar, daß eine porzellanweiße Trübung, die in der gelblich durchscheinenden erstarrten Schmelze von außen nach innen fortschreitet, sich zeigt, - doch keinen Effekt auf der Abkühlungskurve liefern. HÜtTres u. Tammans (8) geben schätzungsweise (bei Benutzung von ca. $25 \mathrm{~g}$ Salz) die Zahlen 200 und 300 bis $200^{\circ}$ bzw. für $\mathrm{K}_{2} \mathrm{MoO}_{4}$ und $\mathrm{K}_{2} \mathrm{WO}_{4}$. AMadori (24) benutzt $30 \mathrm{~g}$ Substanz und findet ganz andere Werte, nämlich 475 und $575^{\circ}$, welche, wie sich herausstellte, völlig unrichtig sind.

Für die Darstellung des Molybdats, worüber die genannten 
Autoren nichts Näheres angeben, wurde ausgegangen von reinem Kaliumcarbonat und von Molybdäntrioxyd (de Haën) das, nach Mercks Angaben geprüft, keine merklichen Verunreinigungen enthielt; auch Sulfat war nicht nachzuweisen. Die getrockneten Präparate wurden in berechneten Quantitäten gemischt und im Platintiegel vorsichtig erhitzt bis zur eintretenden Sinterung; darauf wurde die Temperatur bis zur Schmelzung gesteigert. Das gesinterte, sowie das unvollkommen geschmolzene Produkt zeigen die merkwürdige Erscheinung des Zerrieselns während der Abkühlung, was mit dem Kohlensäuregehalt der Substanz zusammenhängt. Denn wenn alle Kohlensäure ausgetrieben ist, so bildet das erstarrte Salz eine bleibend zusammenhängende Masse. Die Analyse (nach Fresenios I, 6. Aufl., S. 378) ergab: $\mathrm{MoO}_{3}$ gef. $60.6 \%$; ber. $60.46 \%$.

Die Darstellung aus wässeriger Lösung wurde ebenfalls versucht, um durch Umkristallisieren ein ganz reines wasserfreies Salz darzustellen; dieses gelingt aber kaum wegen der großen Zerfließlichkeit der entstehenden, leicht löslichen Kristalle.

Die thermische Untersuchung führte zu dem Resultat, daB dieses Salz tetramorph ist. Die erhaltenen Zahlen in M.V. sind in der folgenden Tabelle zusammengestellt.

Tabelle 9.

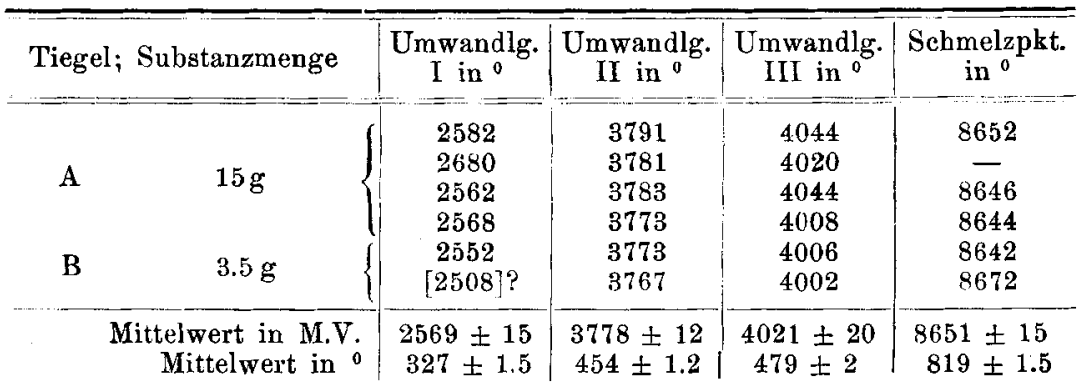

Die Tabelle zeigt, daB die extremen Werte weiter auseinander liegen als bei den schon besprochenen Salzen. Das liegt natürlich einerseits an der nicht vollkommenen Reinheit des Präparats, andererseits auch an der Kleinheit der thermischen Effekte, die bei Umwandlungspunkten II und III tatsächlich sehr wenig ausgesprochen sind. Weiter ist ersichtlich, daB die Angabe Amadoris (475 ${ }^{\circ}$ sich auf den Umwandlungspunkt III beziehen muB; die thermisch wichtigste, mit bloßem Auge erkennbare Umwandlung bei ca. $327^{\circ}$ ist ihm offenbar entgangen. 
Den Vergleich der Schmelz- und Umwandlungspunkte des Kaliummolybdats bringt die folgende Tabelle.

Tabelle 10.

\begin{tabular}{|c|c|c|c|c|}
\hline Beobachter & $\begin{array}{l}\text { Umwandlg. } \\
I \text { in }{ }^{0}\end{array}$ & $\begin{array}{l}\text { Umwandlg. } \\
\text { II in }{ }^{\circ}\end{array}$ & $\begin{array}{l}\text { Umwandlg. } \\
\text { III in }\end{array}$ & $\underset{\text { in }}{\text { Schmelzpkt. }}$ \\
\hline 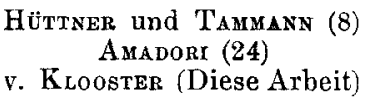 & $\begin{array}{l}200 ? \\
327\end{array}$ & $\overline{454}$ & $\begin{array}{l}\overline{475} \\
479\end{array}$ & $\begin{array}{l}926 \\
926 \\
919\end{array}$ \\
\hline
\end{tabular}

$\mathbf{K}_{2} \mathbf{W O}_{4}$. - Die Darstellung des Salzes geschah wieder auf trockenem Wege, weil das Wolframat noch zerflieBlicher ist als das Molybdat. Außer dem reinen Kaliumcarbonat wurde benutzt Wolframtrioxyd, purissimum, von de Haën, das abgesehen von adsorbiertem Wasser (durch Ausglühen entfernt) nur 0.05\% Eisen enthielt. Der Gewichtsverlust durch Sinterung und nachheriges Schmelzen entsprach ziemlich genau dem Kohlensäuregehalt. Eine $\mathrm{WO}_{3}$-Bestimmung nach von KNorRe (Treadwell 2, 5. Aufl. S. 242) gab $71.4 \%$; ber. $71.1 \%$.

Im Tiegel A wurden ca. $15 \mathrm{~g}$, im Tiegel B $4 \mathrm{~g}$ Substanz untersucht. Die Resultate, mit diesen beiden Mengen Salz erhalten, gaben wieder eine befriedigende Übereinstimmung. Der Schmelzpunkt wurde nach beiden. Methoden wiederholt bestimmt und bei $8686 \pm$ $8 \mathrm{M} . \mathrm{V}$. oder $921^{\circ} \pm 1^{\circ}$ gefunden. Die Erstarrung, die meist nach einer Unterkühlung von $4-5^{\circ}$ eintrat, erfolgte höchstens $1-2^{\circ}$ unterhalb des Schmelzpunktes.

Eine Umwandlung mit deutlich sichtbarem Effekt, liegt bei ca. $388^{\circ}$. Der Effekt wird, besonders bei erstmaligem Erhitzen, leicht verschleppt, oder es tritt eine Überhitzung (von etwa $0.5-1^{\circ}$ ) ein. Merkwürdig ist auch, daß diese Umwandlung weitgehend unterkühlt werden kann. Bei der Abkühlung bis $285^{\circ}$ blieb die Umwandlung aus, wie auch aus der nachfolgenden Erhitzungskurve (bis $420^{\circ}$ aufgenommen) ersichtlich war. Erneute Abkühlung bis $220^{\circ}$ war wieder erfolglos. Darauf wurde schnell gekühlt bis auf etwa $50^{\circ}$, durch Herausnehmen des Tiegels (B) aus dem Ofen und dann von neuem erhitzt, worauf die Umwandlung wieder in der Nähe von $388^{\circ}$ auftrat. Diese Umwandlung wird, wie schon ron HütTner und TammanN $(8,1$. c. S. 227) richtig bemerkt worden ist, bei der Abkühlung von einer merklichen Ausdehnung begleitet. Bei einem Versuch hatte nämlich der Tiegel ein kleines Loch erhalten, durch welches die Schmelze teilweise ausgeflossen war; nach der 
Erstarrung löste sich das Salz als feines Pulver von der Tiegeiwandung.

Zum SchluB sei noch bemerkt, daB bei den Versuchen mit dem Tiegel A eine geringe (bei Benutzung des Tiegels B kaum erkennbare) Wärmetönung bei etwa $457^{\circ}$ auftrat, die nicht mit Sicherheit als eine Umwandlung qualifiziert werden konnte und deshalb in der folgenden Tabelle weggelassen wurde.

Tabelle 11.

\begin{tabular}{c|c|c} 
Beobachter & Unwandlung in & Schmelzpunkt in \\
\hline HÜTrNER und TAMMAN (8) & $300-200(?)$ & 926 (906? l. e. S. 225) \\
AMADORI (24) & 575 & 894 \\
VAN KLOOSTER (Diese Arbeit) & 388 & 921
\end{tabular}

Die Abkühlungsmethode versagt in diesem Fall vollständig, die Angabe AMADORIS $\left(575^{\circ}\right)$ dürfte sich auf eine eutektische Abscheidung beziehen, da ja auch der Schmelzpunkt bedeutend tiefer liegt.

\section{Zusammenfassung.}

Fassen wir das Resultat dieser Untersuchung noch einmal kurz zusammen, so läßt sich feststellen, daß

1. bei genauer Regulierung der Erhitzungsgeschwindigkeit sind - sowohl mit kleinen Mengen von $1 \mathrm{qcm}$, als mit größeren von $5 \mathrm{qcm}$ und mehr - die Schmelz- und Umwandlungspunkte bis auf etwa $1^{0}$ genau reproduzierbar sind.

2. die Unzulänglichkeit der Abkühlungsmethode, namentlich im Fall, daß Umwandlungen auftreten, au einigen Beispielen überzeugend nachgewiesen ist.

3. sich von den untersuchten Salzen, außer dem reinen $\mathrm{Na}_{2} \mathrm{SO}_{4}$, das $\mathrm{Na}_{8} \mathrm{WO}_{4}$ und das $\mathrm{K}_{2} \mathrm{SO}_{4}$, die beide in reinem Zustande leicht $\mathrm{zu}$ gänglich sind, sehr gut als Eichungsobjekte verwerten lassen. Zwar haben DAY und Sosman (1, S. 84) Metalle angewendet, hauptsächlich, weil dies allgemein üblich ist; aber mit vollkommen reinen Salzen sind die Schmelżpunkte fast ebenso scharf und stimmen auch die Erstarrungspunkte (eine wichtige Kontrolle für die richtige Wahl der benutzten Versuchsanordnung) vollkommen damit überein, während keine Gefahr für Infektion der Thermoelemente vorliegt. Auch könnte man hier noch eventuell die Umwandlungstemperaturen als weitere Fixpunkte verwenden.

4. die Schmelz- und Umwandlungstemperaturen bestimmt wurden wie in der folgenden Tabelle angegeben. 
Tabelle 12.

\begin{tabular}{c|c|c|c|c}
\hline \hline $\mathrm{Salz}$ & $\begin{array}{c}\text { Umwandlg. I } \\
\text { in }\end{array}$ & $\begin{array}{c}\text { Umwandlg. II } \\
\text { in }\end{array}$ & $\begin{array}{c}\text { Umwand)g. III } \\
\text { in }\end{array}$ & $\begin{array}{c}\text { Schmelzpunkt } \\
\text { in }\end{array}$ \\
\hline $\mathrm{Na}_{8} \mathrm{SO}_{4}$ & 241 & - & - & 884 \\
$\mathrm{Na}_{2} \mathrm{MoO}_{4}$ & 445 & 592 & -640 & 687 \\
$\mathrm{Na}_{2} \mathrm{WO}_{4}$ & 587 & - & - & 694 \\
$\mathrm{~K}_{2} \mathrm{SO}_{4}$ & - & 582 & - & 1067 \\
$\mathrm{~K}_{2} \mathrm{MoO}_{4}$ & 327 & 454 & 479 & 919 \\
$\mathrm{~K}_{2} \mathrm{WO}_{4}$ & 388 & - & - & 921
\end{tabular}

Aus der Tabelle ist schlieBlich noch ersichtlich, daB sowohl bei den Na- als bei den K-Salzen die Schmelztemperatur vom Sulfat bis zum Wolframat ein Minimum beim Molybdat zeigen, während z. B. bei den drei Na-Salzen die Umwandlungstemperaturen I gleichmäBig ansteigen. Die Umwandlung des $\mathrm{K}_{2} \mathrm{SO}_{4}$ bei $582^{\circ}$ würde daher wohl, aller Wahrscheinlichkeit nach, dem Typus II bei den anderen Salzen entsprechen.

Literaturzusammenstellung betreffs den Schmelz- und Umwandlungspunkten der untersuchten Salze.

1) Carnegie Inst. of Washington. Publ. 1557 (1911).

2) Journ. of the Wash. Ac. of Sc. 1 (1911), 49.

3) Americ. Journ. Sci. [4] 28 (1909), 453.

4) Americ. Journ. Sei [4] 31 (1910), 341.

5) Ann. d. Phys. [3] 55 (1895), 95.

6) Ann. des Mines [9] 11 (1897), 209.

7) Berl. Ber. 36 (1903), 2357.

8) Z. anorg. Chem. 43 (1905), 215.

9) Z. anorg. Chem. 50 (1906), 355.

10) Z. f. Elektrochem. 12 (1906), 337.

11) N. Jahrb. f. Min ete., Beil. Bd, 24 (1907), 1.

12) Zeitschr. phys. Chem. 64 (1908), 343.

13) Z. anorg. Chem. 61 (1909), 122.

14) N. Jahrb. f. Min. etc., Beil. Bd. 30 (1910), 1.

15) Rend. Ace. lincei [5] 19II (1910), 422.

16) Rend. Ace. lincei [5] 21 II (1912), 65.

17) Z. anorg. Chem. 58 (1908), 113.

18) Gazz. chim. ital. 39 II (1909), 55.

19) Z. anorg. Chem. 69 (1910), 135.

20) Bull. soe. min. de France 5 (1884), 226.

21) Bull. soe. chim. 47 (1887), 300.

22) Centrbl. f. Min. (1909), 728.

23) Rend. Acc. lincei [5] 21 II (1912), 71.

24) Rend. Ace. Lincei [5] 22 II (1913), 453.

Groningen (Holl.), Anorg. Chem. Lab. der Reichsuniversität, September 1913.

Bei der Redaktion eingegangen am 7. November 1913. 